\title{
Evaluating Tire Pressure Monitoring System for Traffic Management Purposes - Simulation study
}

\author{
Nemanja Savić ${ }^{1}$, Marek Junghans ${ }^{2}$ and Miloš Krstić ${ }^{3}$
}

\begin{abstract}
In this paper, we evaluate Tire Pressure Monitoring System (TPMS) for traffic management purposes. It has been shown that up to $60 \%$ of the vehicles can be detected in urban traffic environments, which makes it suitable for deriving: routes, travel times and the traffic state. In particular, the theoretical background and basic concepts are given. Furthermore, we present a simple simulation model of TPMS based on empirical investigations. A simulation platform, based on traffic simulator, used for evaluation is introduced. Next, simulation results related to the number of detected vehicles are given regarding detection range, sensor transmission period and traffic flow. The impact of the roadside unit's location, as well as the number of detected vehicles, is investigated by simulating a realistic traffic scenario. Finally, the applicability of TPMS for deriving different traffic information is evaluated.
\end{abstract}

\section{INTRODUCTION}

Modern trends in collecting data from the traffic strive towards finding novel approaches and technologies able to provide lower implementation costs and high quality information needed for an efficient, environmentally friendly and safe traffic and transportation system. Traffic detection technologies can be divided into two groups: technologies suitable only for vehicle counting, and technologies suitable for vehicle identification. Detection technologies, such as inductive loops, radars, laser scanners, etc. are traditionally used for vehicle counting purposes. However, vehicle counting is insufficient when, for example, travel times, OD matrices and information about routes are required. In order to extract this information which is essential for traffic management, it is necessary to use technologies that are able to provide vehicle identification and re-identification.

Technologies most often used for vehicle identification are: ANPR (Automatic Number Plate Recognition), RFID (Radio-frequency identification), Bluetooh, WiFi and GSM (Global System for Mobile Communications). ANPR is the typical example for a direct vehicle identification technology. It relies on detecting the only unique and visible vehicle identifier, i.e. the plate number, and is widely used on highways for automatic toll collection, travel time measurements and for other traffic surveillance applications. The main disadvantage of ANPR comes from privacy concerns due to the direct link between plate numbers and car owners. The performance

\footnotetext{
${ }^{1}$ Nemanja Savić is with DLR (German Aerospace Center), Institute of Transportation Systems, Rutherfordstr. 2, 12489 Berlin, Germany nemanja.savicedlr.de

${ }^{2}$ Marek Junghans is with DLR (German Aerospace Center), Institute of Transportation Systems, Rutherfordstr. 2, 12489 Berlin, Germany marek. junghans@dlr.de

${ }^{2}$ Miloš Krstić is with IHP, Im Technologiepark 2, 15236 Frankfurt, Germany krsticdihp-microelectronics.com
}

of ANPR can be negatively influenced by weather conditions (heavy rain, snow or fog) and spacing between vehicles which can disturb the line of sight. Currently employed ANPR cameras can achieve recognition accuracy from $90 \%$ up to $98 \%$ [1], [2], [3], [4].

Other technologies, such as Bluetooth, GSM and WiFi, can be used for indirect vehicle detection. That means that the vehicle is not necessarily equipped with the particular device, but it is highly possible to detect the device carried by the driver or other passengers. Indirect Bluetooth detection is already in commercial use and there are a number of solutions available on the market. Indirect WiFi detection is still under investigation, and several studies in this field have been published, e.g. [5]. Indirect detection relies on pervasive use of smart devices. These devices are usually equipped with one or more wireless communication interfaces, such as Bluetooth or WiFi. During their operation, a certain ID (MAC address or similar) is transmitted, which makes device identification possible, but also vehicle identification with the assumption that the device was inside the vehicle. Indirect detection might suffer from an error due to detection of devices belonging to the pedestrians or cyclists, which is the case in highly pedestrianized areas [6].

Modern vehicles have a significant number of installed sensors used for measuring vital parameters, such as speed, temperature, gear positioning, cruise control setting, gasoline level, etc. These sensors are connected to an ECU (Electronic Control Unit) using wired connections, which entails the use a few miles of wire per car for such purposes. In order to reduce the wire length and thus the weight of the vehicle, there is significant interest among car manufacturers for using wireless connections instead of wired ones [7], [8]. The nature of in-car wireless networks makes them vulnerable to eavesdropping and possible misuse, but also makes them suitable for direct vehicle detection. One example of using incar wireless networks is TPMS, used for collecting pressure values from pneumatics. Besides its primary use, TPMS can alternatively be used for collecting traffic data. This can be accomplished by receiving TPMS signals externally and using their information for vehicles identification.

The main goals of this study are: (1) evaluation of achievable detection ratios of the vehicles using TPMS, and (2) discovering the consequences of the achieved detection ratios on possible utilization for deriving traffic information. In section 2 , the basic concepts of TPMS are given, whilst in section 3 , we present the idea of using TPMS for collecting traffic data. At this point, the theoretical background, as well as the evidence for the concept, will be shown. The evaluation of 
the concept is accomplished by using a simulation approach. The simulation platform will be presented in section 4 . In section 5, we present the results of the simulation of two traffic scenarios. The conclusion and future prospects are given in section 6 .

\section{TPMS (TIRE Pressure MONitORing SyStem)}

TPMS is an electronic system used for monitoring the air pressure inside pneumatic tires. The real time information about pressure values is available to the driver via various indicators. In order to improve traffic safety, many countries declared TPMS mandatory through legislation. In the US, installation of TPMS is required for new vehicle types since 2007, while in the EU, starting from November 1, 2014, all new passenger vehicles must be equipped with TPMS. In many other countries, South Korea, China, Japan, etc. TPMS will also be mandatory in the future [9].

There are two common methods currently in use for monitoring tire pressure: indirect (iTPMS) and direct (dTPMS). iTPMS does not perform any wireless data exchange and cannot be used for traffic data detection purposes, thus we will only focus on direct TPMS.

Direct TPMS employs sensors specifically designed for the purpose of measuring pressure and other important parameters inside the tire. In comparison to iTPMS, dTPMS has sensors installed inside the tires or mounted on the valves. The sensors measure the absolute tire pressure value. The measured parameters are transmitted over radio link to the controlling unit (ECU) placed inside the vehicle. A typical TPMS frame format consists of the following fields: preamble, ID, pressure, temperature, error correction code, and other status and error correction data [10]. The radio link for European market sensors lies in the $434 \mathrm{MHz}$ ISM band, while in the US the $315 \mathrm{MHz}$ band is also allowed to be used. Upon reception of the signal, the ECU processes it and extracts the information which later shown on the driver's front panel. The structure of direct TPMS is shown in figure 1.

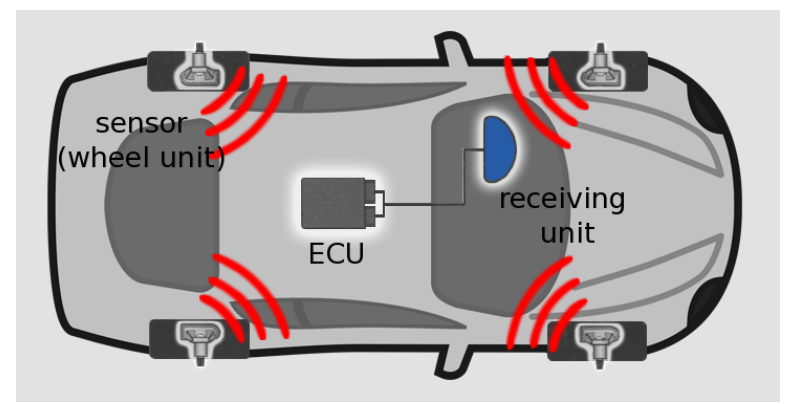

Fig. 1. Direct TPMS structure. Picture edited from [9].

\section{UTILIZING TPMS FOR DERIVING TRAFFIC DATA}

Since TPMS sensors transmit their data wirelessly, a complete new spectrum of alternative TPMS utilization is possible. Receiving TPMS frames externally and extracting ID allows us to use TPMS for vehicle identification, and afterwards for deriving traffic information. This idea is described in [10]. In the following, we will explain the basic concept and present an experimental setup for evaluation of TPMS for collecting traffic data.

\section{A. Basic concept}

The TPMS sensor ID is a 32 bit number contained in the TPMS message frame, used mainly for recognizing sensors belonging to the same vehicle. The length of 32 bit leads to $2^{32}$ possible IDs. If we take into account the maximum life of the sensor (around 10 years, but usually less), trends in the annual vehicle production, different market zones and the number of possible IDs, there is very low probability of having two identical IDs at the same time on the same road. The information about pressure and temperature inside the tires could also be collected and used further for deriving various statistics. Additionally, the information about velocity can be extracted since certain sensor types include this information in their message [9]. The future system for deriving traffic information based on collected TPMS frames would consist of roadside units, i.e. the receivers, able to receive TPMS signals from various sensor types to extract their data and to send them to a data collecting center or traffic management system. Deriving traffic information can be further improved by combining TPMS data and the data from other detection technologies such as WiFi and Bluetooth.

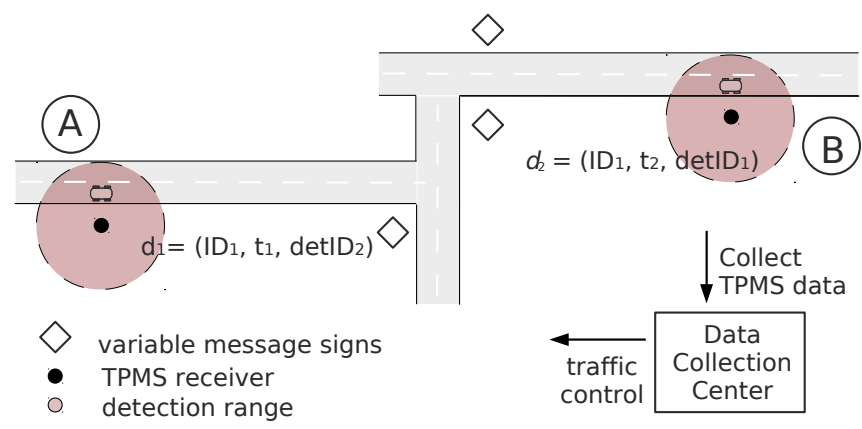

Fig. 2. Traffic data detection using TPMS ([10]).

As mentioned in [10] and [11], there are several benefits of using TPMS for collecting traffic data. Since the communication protocols used in TPMS are quite simple, the receiver can be implemented using off the shelf devices. Due to the legislation, TPMS will be mandatory in Europe, and it is possible to assume that the direct approach will dominate the TPMS market in the future, because of its technical superiority and higher measuring capability comparing to the indirect approach. Hence, we expect that high percentage of the vehicles equipped with dTPMS will be use in 10 to 15 years from now, leading to a significant fleet of detectable vehicles without additional in-car hardware required and even without sophisticated infrastructure. Using TPMS detection, we expect to be able to measure travel times, to extract OD matrices and to detect jam situations. Additionally, we assume to be able to distinguish between passenger vehicles and goods vehicles by reading the pressure value from the 
sensor's message [12]. Bluetooth and WiFi do not offer such possibilities. In this case it is possible to avoid an error in deriving average travel time, which is introduced due to detecting devices from goods vehicles [13]. The negative influence of weather conditions such as snow, rain or fog, which affect ANPR is drastically reduced since TPMS detection is based on receiving radio signals. Unlike the license plate numbers, sensor IDs are not directly linked to the individuals through law enforcement databases, thus reducing the privacy concerns existing with the ANPR. In order to meet various privacy protection regulations, anonymization techniques need to be utilized, in the same manner as with Bluetooth detection.

Besides specified advantages, there are however several disadvantages, which makes the realization of TPMS for traffic data detection challenging. First of all, TPMS communication is not standardized, which leads to numerous implementations of TPMS based on the the demands of the producer itself. This means that future system needs to be able to detect all possible sensor types. However, certain efforts for standardizing this communication exist [9]. Next, EMC (Electromagnetic Compatibility) regulations for vehicles allow sensors to radiate relatively low power level. The power of the transmitted signal is additionally influenced by the metal rim and the car body [14], [15]. Further, the characteristics of the communication channel change dynamically due to the rotation of the wheel and put serious demands on the dynamic range of the receiver [16]. The system should be able to cope with highly redundant data, present due to the fact that every vehicle has more than one TPMS sensor. The scope of this paper will be oriented more towards evaluating potentials for using TPMS in deriving traffic information, while the other addressed problems will be the topics of further research.

Rouf et al. in [11] have shown that TPMS signals could be received from a static TPMS sensor at the distance of approximately $40 \mathrm{~m}$, but also from the sensor in motion at the speed of $35 \mathrm{~km} / \mathrm{h}$. Based on available information about TPMS sensors ([10], [9], [11]) and our recent study, we designed a prototype receiver based on software radio approach. For this purpose, a USRP device from Ettus Research [17] was used as radio frontend, while the software is based on the GNU Radio framework [18]. The range of our receiver is around $100 \mathrm{~m}$. The experiment was conducted on DLR test track, UTraLab - Urban Traffic Research Laboratory [19], in Berlin. Two identical receivers were installed on two gantries at the distance of $850 \mathrm{~m}$. Finally, we were able to derive travel times using TPMS. The results are presented in [20].

\section{TPMS Simulation Model}

The main objective of this paper is to make an initial study of TPMS for traffic management purposes. The initial study is accomplished via simulation. In this way we are able to understand the influence of significant parameters like: equipment rate, detection range, road type and sensor type on the detection performance. The simulation platform is based on the SUMO (Simulation of Urban MObility, [21]) traffic simulator and two other components: a functional model of TPMS (transmitting part) and a model of TPMS signal reception (receiving part). Finally, by using this simulation model, it is possible to evaluate the amount of TPMS frames available within certain areas, which can be considered as the best case approach.

\section{A. Functional model of TPMS}

The functional model of TPMS describes the basic properties and operation of TPMS system in the vehicle. This model is based on simplified TPMS where sensors transmit their signals periodically without being externally triggered. The following assumptions concerning vehicles equipped with TPMS were made:

- Every tire is equipped with TPMS sensor

- All vehicles use the same sensor type

- The sensor ID is unique

- There is no pressure loss in tires and all tires have nominal pressure value

The main parameters of every sensor are:

- sensor ID (4 bytes), ID

- transmission period, $T$

- initial moment of transmission, $t_{\text {init }}$

According to the various sensors documentation [22], and [9], [10], [11], the message transmission period is usually $30 \mathrm{~s}$ to $60 \mathrm{~s}$. We have assumed all sensors have the same transmission period. Sensor transmission is modeled as a stochastic process and every sensor has attached a random number from range $[0$, transmission period), for defining the initial moment of transmission. Transmission can occur only at integer multiples of transmission period after the initial moment.

\section{B. Model of TPMS signal reception}

After modeling the operation of TPMS, the model of reception, i.e. roadside unit, has been designed. Roadside units are receivers usually installed along the road or at the junctions, and they are used for receiving the signals sent by TPMS sensors. Presently, the model of reception is simplified since our current goal is to estimate the amount of sensors transmitting inside the certain area. The detection range, $r$, is the main parameter of the roadside units and determines the maximum distance to the transmitting sensor for correct frame reception. Once the sensor is inside the detection range, the roadside unit examines whether transmission occurs or not, based on the sensor transmission period and initial moment of transmission. If the transmission occurs, there are several parameters which can affect the probability of correct frame reception: velocity of the vehicle, surrounding vehicles placement, physical obstructions, interference, etc. In the current model, only the model of interference is implemented, while the influence of other parameters will be thoroughly investigated in future work. The interference might occur when multiple TPMS sensors transmit in the same time. The outcome of this is difficult to predict, and mostly depends on the distance between interferer and the receiver. 


\section{Simulation platform implementation}

As stated earlier, our simulation platform is based on SUMO. SUMO is an open source, microscopic, inter- and multi-modal, space-continuous and time-discrete traffic flow simulation platform developed by DLR. It contains APIs for interaction with external application via module called "TraCI" - "Traffic Control Interface" [23]. TraCI allows controlling simulation and gathering information about vehicles, traffic lights, and other traffic participants, between two adjacent simulation steps. For implementing the simulation platform we used Python programming language.

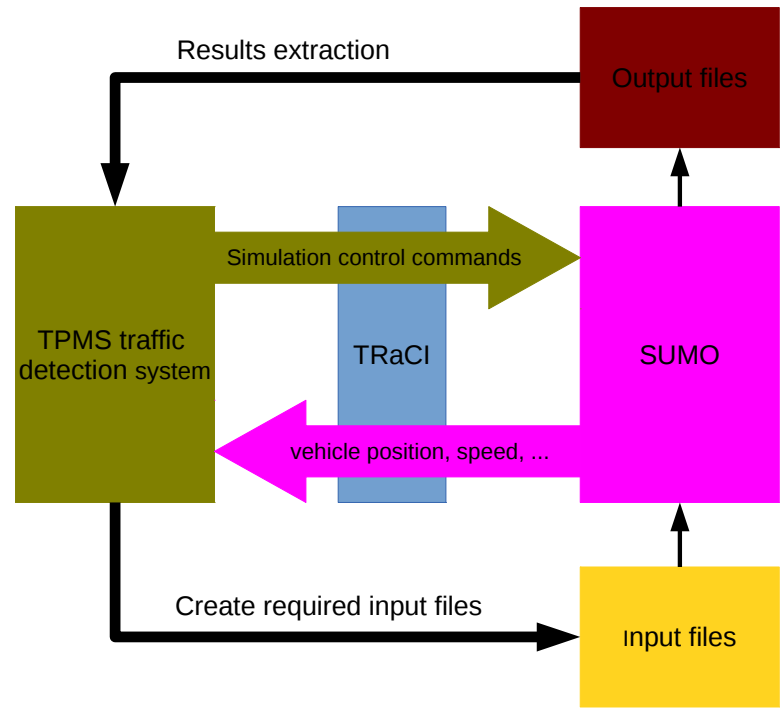

Fig. 3. Simulation platform diagram

The top level program used for performing the simulation consists of two main instances: SUMO process and TPMS traffic detection system, as shown in figure 3 . The SUMO process is responsible for simulating traffic scenarios, while being controlled by the top level code via TraCI. The TPMS traffic detection system implements both TPMS and roadside unit models. Its task on the one hand is to assign sensor IDs to the vehicles coming into scenario, and on the other hand, checking for the transmission within the range of the detectors. A screenshot from the SUMO GUI with the main simulation parameters is shown in figure 4

\section{Simulation RESUlTS}

The evaluation of TPMS for traffic data detection is accomplished through the simulations of two distinct traffic scenarios. The first scenario can be considered as benchmark used for generating referent traffic environment considering traffic flow and density. The second scenario is based on urban traffic model and is used for generating more complex and realistic traffic stimuli. The main parameters for evaluating the number of available TPMS frames inside the range of the receiver are:

- Detection range, $r$

- TPMS sensor transmission period, $T$

- Travel speed of the vehicle, $V_{\text {avg }}$

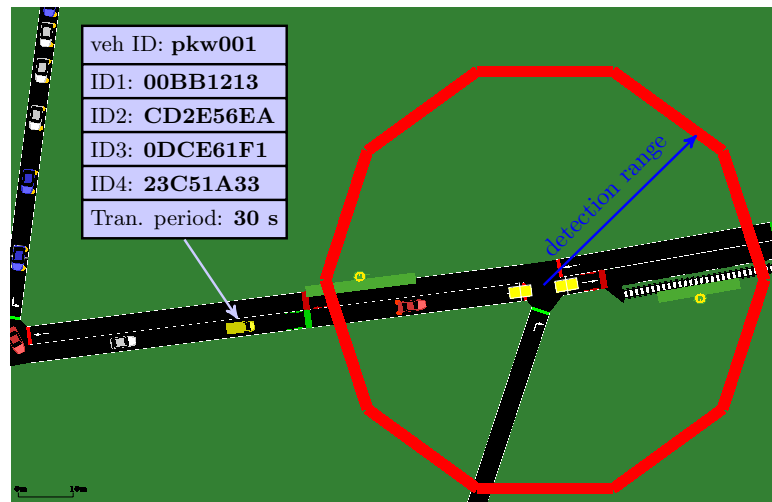

Fig. 4. A screenshot from SUMO GUI with the main model parameters.

The analytical approach shows that probability of transmission occurrence within the range is proportional to the ratio of the detection range and the distance traveled between two adjacent transmissions, i.e. the distance traveled during sensor transmission period. The ratio is defined as:

$$
p=\frac{r}{V_{\mathrm{avg}} \cdot T}
$$

As can be seen, the number of available frames can be increased either by improving the receiver's range, or by ensuring a longer time of stay within the range. To achieve this, the detector can be placed along the road with lower allowed speed or near a traffic light, while the equipment ratio is assumed to be constant.

In order to quantitatively express performance of the TPMS based traffic data detection approach, we defined two performance indicators. The indicators show relative number of detections in predefined time interval (i.e. aggregation period) and they are defined as:

- sensors detection ratio, $d_{\mathrm{s}}$, as the ratio of the number of successfully received TPMS frames, $n_{\text {detsens }}$, and the total number of sensors present inside the range, $n_{\text {totsens }}$, within observed time interval, i.e. aggregation period:

$$
d_{\mathrm{s}}= \begin{cases}\frac{n_{\text {detsens }}}{n_{\text {totsens }}} & \text { if } n_{\text {totsens }}>0 \\ 0 & \text { if } n_{\text {totsens }}=0\end{cases}
$$

- vehicles detection ratio, $d_{\mathrm{v}}$, as the ratio of the number of vehicles whose sensor(s) were detected, $n_{\text {detveh }}$, and the total number of vehicles present inside the range, $n_{\text {totveh }}$, within observed time interval, i.e. aggregation period:

$$
d_{\mathrm{v}}= \begin{cases}\frac{n_{\text {detveh }}}{n_{\text {totveh }}} & \text { if } n_{\text {totveh }}>0 \\ 0 & \text { if } n_{\text {totveh }}=0\end{cases}
$$

For defining vehicles detection ratio we assumed that the monitoring system will be able to group sensors, which belong to the same vehicle, thus significantly improving identification of the vehicles.

In our simulations we assumed equipment ratio of $100 \%$, and the results for lower equipment ratios can be obtained by scaling down given results. The second assumption is that 
every vehicle has 4 tires, thus the ratio of $d_{\mathrm{v}}$ and $d_{\mathrm{s}}$ can be between zero and four. A value which is smaller than 1 is possible if the equipment ratio is less than $100 \%$.

\section{A. Scenario 1 - Benchmark scenario}

The first scenario was used for generating artificial traffic environment. It consists of a simple circular lane with the length dependent on following predefined parameters: traffic density and the number of vehicles. When the simulation starts, the speed of the vehicles is adjusted based on the density and a car-following model (Krauß model [24]). Before the simulation starts, we specify start and end density values, position and range of the detectors and the aggregation period. The simulation was then run incrementally for the density values between the given interval. At the end of the simulation, $d_{\mathrm{s}}$ and $d_{\mathrm{v}}$ are derived.

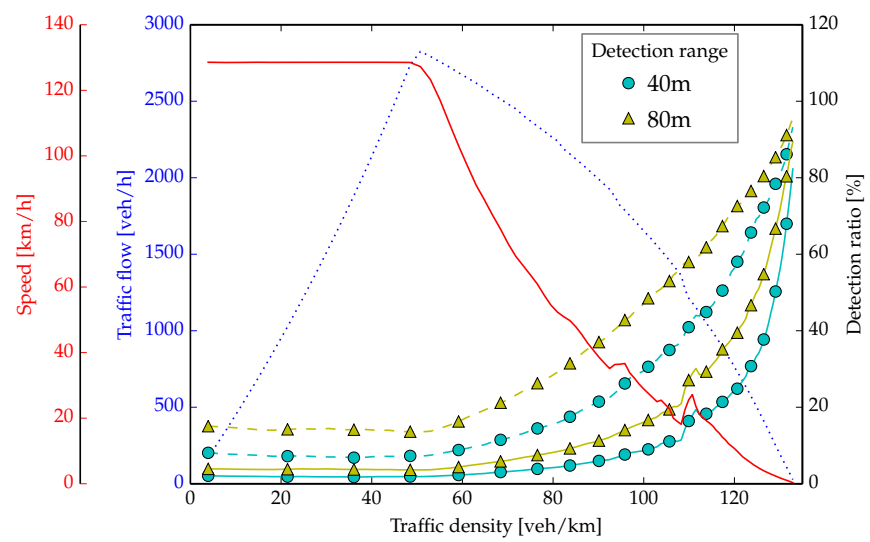

Fig. 5. Sensors detection ratio (solid line) and vehicles detection ratio (dashed line) vs. traffic density for transmission period of $60 \mathrm{~s}$ and detection range as parameter. The aggregation period is $120 \mathrm{~s}$.

Figure 5 shows the average values of the sensors detection ratio and vehicles detection ratio as a function of traffic density. The transmission period, $T$, was set to $60 \mathrm{~s}$. Additionally, for the sake of clarity, traffic flow (dotted blue line) and the average speed (solid red curve) at the observed lane are also plotted in figure 5. In the case of free flow (i.e. for traffic density less than $50 \mathrm{veh} / \mathrm{km}$, and speed about $130 \mathrm{~km} / \mathrm{h}$, both sensors and vehicles detection ratios are almost constant and depend mainly on the maximum allowed speed on the road and the detection range. In other words, the visibility time (the time of stay inside the range) is constant and so are the detection ratios. One can realize that the vehicles detection ratio is between 3.5 and 4 times higher than the sensors detection ratio. This is due to the fact that only a few detected sensors belong to the same vehicle. At a density of around $50 \mathrm{veh} / \mathrm{km}$, the traffic breakdown is about to begin. As the effect of density rise, the speed of the vehicles is reduced, thus the time of stay within the range is higher, which yields the increase of the number of detected sensors and vehicles. The ratio between detected vehicles and detected sensors starts to drop because the number of detected sensors per vehicle rises. In the area of unstable traffic, for density between $80 \mathrm{veh} / \mathrm{km}$ and $110 \mathrm{veh} / \mathrm{km}$, there are some fluctuations of the sensor detection ratio, which we believe is due to the variation of speed, but the trend of increase in detection ratios is still present. When the speed drops to $10 \mathrm{~km} / \mathrm{h}$, which happens for density values above $120 \mathrm{veh} / \mathrm{km}$, between $20 \%$ and $40 \%$ of the sensors are detected (depending on the detection range) which is $60 \%$ to $80 \%$ of detected vehicles. Finally, at traffic densities above $130 \mathrm{veh} / \mathrm{km}$, the stop-and-go effect in traffic appears and nearly all vehicles are detected.

As can be seen, the number of detected vehicles is highly influenced by the speed and detection range. If higher detection ratios are required, then suitable place for the receivers would be roads with low speed limits or traffic lights. On the other hand, for speed measurement on highways, quite wide coverage is required in order to ensure frame reception. If the goal is traffic monitoring in urban areas, then traffic lights could be very suitable positions for the receivers in order to improve the detection ratio. Additionally, TPMS receivers can be installed at the places where traffic jams are expected to occur, for detecting such conditions. As for detection range, we expect obtainable values of up to $100 \mathrm{~m}$. Figure 5 shows that doubling the detection range yields doubling the detection ratio, but as stated before, a long detection range can introduce an error in certain applications.

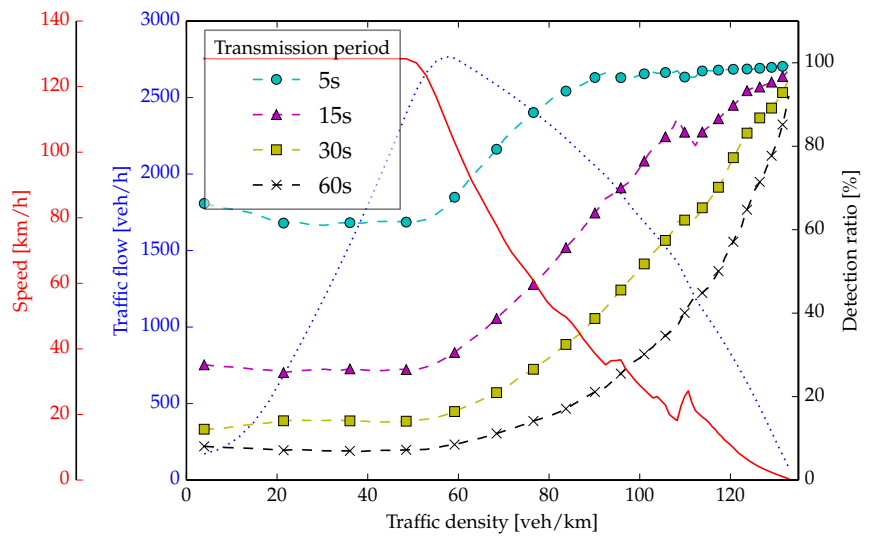

Fig. 6. Vehicles detection ratio vs. traffic density for different transmission period values, detection range of $40 \mathrm{~m}$ and aggregation period of $120 \mathrm{~s}$.

Another parameter that influences the detection ratio is the sensor type. Sensors can differ from each other by transmission period, number of frames per message and the radiated power. Here, we assumed that sensors transmit one frame per message and radiate with identical power. Figure 6 shows the average detection ratio of the vehicles as a function of traffic density and transmission period. The chosen values for the transmission period could be found in different types of TPMS sensors currently available on the market. The probability of detecting sensors with lower transmission period is higher compared to sensors with longer transmission period within the same time interval. As can be seen in figure 6 , the detection ratio reaches values of more than $60 \%$ even for higher speeds when the transmission period is $5 \mathrm{~s}$. For traffic density above $80 \mathrm{veh} / \mathrm{km}$, and speed less than $50 \mathrm{~km} / \mathrm{h}$, more than $90 \%$ of the vehicles are detected. In one of our 
experiments that we conducted on the test track, sensors with a $5 \mathrm{~s}$ transmission period were the majority of the detected sensors, which is in accordance with the simulation results [20].

\section{B. Scenario 2 - Acosta}

The second scenario, named Acosta, is based on a traffic model of a section of the city of Bologna in Italy. It contains 9000 vehicles and covers the morning rush hour in the interval from 8 a.m. to 9 a.m. [25]. This scenario consists of several intersections, interconnected with main urban roads, located near the city center. By simulating the Acosta scenario, we will show obtainable detection ratios in urban traffic environment. The assumption is that TPMS will rather be used for traffic monitoring purposes in urban areas due to higher detection ratio, compared to highways.

For the purpose of proving the influence of speed on the detection ratio we made the setup with two detectors, as shown in figure 7. Two receivers, $\mathrm{A}$ and $\mathrm{B}$, with detection range of $50 \mathrm{~m}$, were placed on the same edge for ensuring the same amount of traffic passing them. Receiver A is located at the traffic light, while receiver B was placed $180 \mathrm{~m}$ away. The simulation results are shown in figure 8 . The vehicles detection ratio, $d_{\mathrm{veh}}$ (red curve), average vehicles detection ratio, $d_{\text {vehavg }}$ (black) and average speed, $v_{\text {avg }}$ (blue) are plotted over the course of one hour.

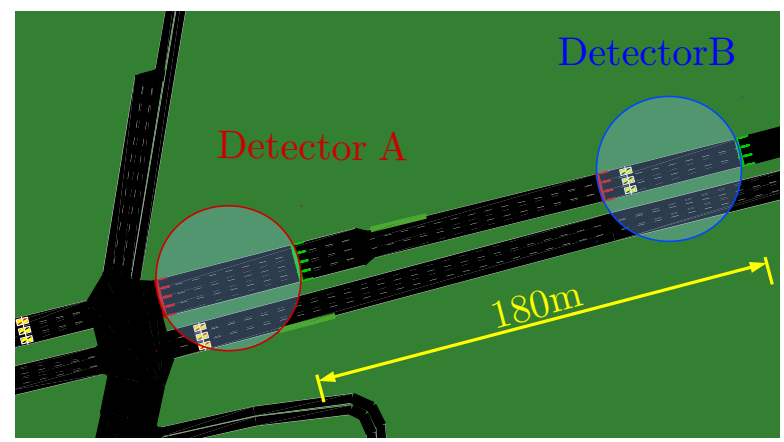

Fig. 7. A setup for showing influence of speed on the detection ratio.

As can be seen, $v_{\text {avg }}$ and $d_{\text {veh }}$ at detector A change dynamically. When the traffic light is red, vehicles are approaching with reduced speed and jamming. Hence, the detection ratio rises up to $80 \%$ for speed values less than $20 \mathrm{~km} / \mathrm{h}$. On the other hand, when the traffic light is green, the speed of the vehicles rises, and therefore the detection ratio decreases to less then $40 \%$ for the speed values up to $40 \mathrm{~km} / \mathrm{h}$. In contrary, at detector $\mathrm{B}$, the detection ratio is almost constant, with the value of $20 \%$. This is due to the fact that the average speed at detector A (about $20 \mathrm{~km} / \mathrm{h}$ ) is lower in comparison to the speed at detector B (about $50 \mathrm{~km} / \mathrm{h}$ ). The gain in vehicles detection ratio introduced by placing detectors at traffic lights is nearly 4 , which is clearly dependent on the speed of the vehicles. As a consequence, if higher detection ratio is needed, TPMS detectors should be installed at the places where the expected average speed of the passing vehicles is low.
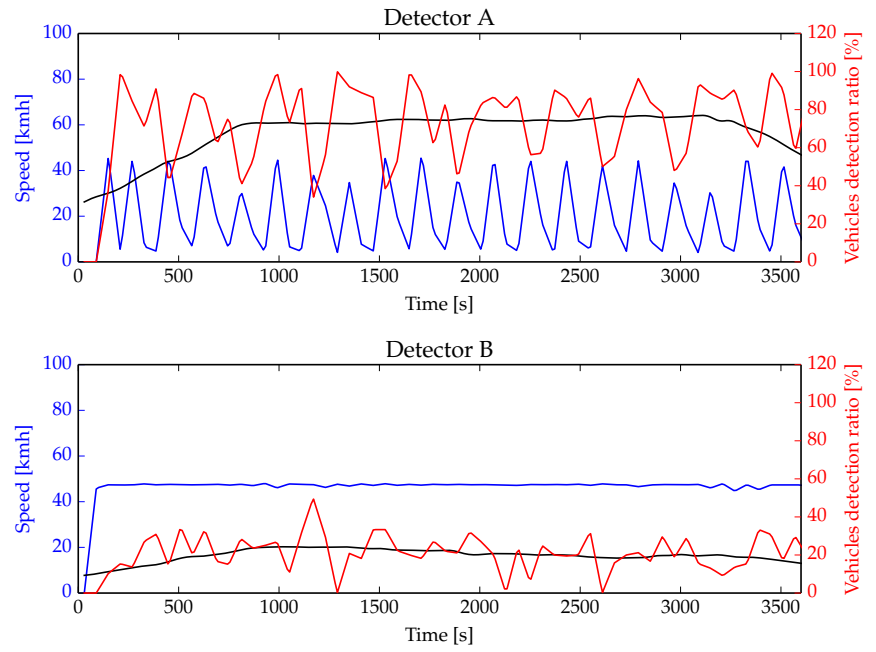

Fig. 8. Comparison of vehicles detection ratio near traffic light (detector A, upper plot) and far from the traffic light (detector B, lower plot). The detection range is $50 \mathrm{~m}$. The transmission period of the sensors is $60 \mathrm{~s}$. The aggregation period is $60 \mathrm{~s}$.

Let us now assume that TPMS will be used for monitoring urban traffic with detectors placed on junctions. Hence, we want to estimate the expected ratio of detected vehicles at the junctions. Therefore, we chose 4 big junctions with traffic lights from the Acosta scenario, and placed our receivers to cover all their incoming links. At the end of the simulation we derived the average vehicles detection ratio of the junction as the average $d_{\text {vehavg }}$ from every link. The results are given in table I]

TABLE I

VEHICLES DETECTION RATIO AT JUNCTIONS

\begin{tabular}{|c|c|c|c|c|}
\hline Junction & 1 & 2 & 3 & 4 \\
\hline$d_{\text {vehavg }}$ & $45 \%$ & $50 \%$ & $52 \%$ & $50 \%$ \\
\hline$N_{\text {vehicles }}$ & 2578 & 3581 & 3898 & 4352 \\
\hline
\end{tabular}

The obtained results are indeed much more than we expected. Nevertheless, depending on the traffic application, this can be more than necessary or even too less: In the case of counting vehicles for an accurate traffic flow measurement or for the detection of accurate time gaps for traffic control the obtained amount of about $50 \%$ is currently too small to achieve good results. Particularly in the case of a highly dynamic environment in a highly instationary traffic process, accurate estimations are almost impossible. For this case the time dependent probability density distributions of the traffic parameters are urgently needed to calibrate the TPMS systems. Nevertheless, some investigations on the basis of FCD (floating car data) have shown a successful realization of traffic control. Indeed, this requires an FCD equipment ratio of more than $20 \%$ [26], [27].

On the other hand, in many cases, traffic engineers are only interested in accurate travel times to obtain a good Level of Service (LoS) in a certain area, e.g. an intersection. For such cases, the obtained detection ratios of about $50 \%$ 
are acceptable. According to some publications ([28], [29]) concerning the equipment ratio of FCD/X-FCD (floating car data/extended FCD) only an amount of about $3 \%$ of the vehicles need to be detected to determine a good LoS and to detect a traffic breakdown within a time window of $10 \mathrm{~min}$.

In the case of transportation planning, there is a great need for determining accurate and time-dependent origindestination (OD) and route data. Currently, such data are acquired manually on the basis of the structure of a city considering the settlement structure. Concerning obtained results, accurate estimation of OD and route data using TPMS will be challenging task.

Altogether, we can conclude, the applicability TPMS for an accurate traffic monitoring or even for traffic management purposes, is quite promising.

\section{Vi. Conclusion and Future Prospects}

In this paper, we evaluated the usability of TPMS for traffic data detection applications. The evaluation is accomplished by simulation. For this purpose a simulation model of TPMS sensors detection is proposed and combined with the SUMO traffic simulator. The results of the benchmark scenario showed that the number of detected vehicles reach $20 \%$ under free flow conditions, while in congested traffic it can reach more than $80 \%$. The second scenario, Acosta, is used to show the impact of the roadside unit location on vehicles detection. Two locations are observed, traffic light and free flow edge. The obtained results show the gain of 4 when the roadside unit is located at the traffic light. Additionally we have shown the average value of the detection ratio at the junctions is about $50 \%$, which is quite acceptable for several traffic applications.

Regarding future work, we will concentrate on improving our simulation model by performing deeper analysis of the communication between roadside unit and moving sensor regarding speed. For this purposes, a prototype receiver will be designed and field measurements on DLR test track in Berlin will be performed.

\section{REFERENCES}

[1] L. Keilthy, "Measuring anpr system performance," Parking Trend International, 2008.

[2] J.-W. Hsieh, S.-H. Yu, and Y.-S. Chen, "Morphology-based license plate detection from complex scenes," in Pattern Recognition, 2002. Proceedings. 16th International Conference on, vol. 3. IEEE, 2002, pp. 176-179.

[3] C.-T. Hsieh, Y.-S. Juan, and K.-M. Hung, "Multiple license plate detection for complex background," in Advanced Information Networking and Applications, 2005. AINA 2005. 19th International Conference on, vol. 2. IEEE, 2005, pp. 389-392.

[4] J. Matas and K. Zimmermann, "Unconstrained licence plate and text localization and recognition," in Intelligent Transportation Systems, 2005. Proceedings. 2005 IEEE. IEEE, 2005, pp. 225-230.

[5] A. Luber, M. Junghans, S. Bauer, and J. Schulz, "On measuring traffic with wi-fi and bluetooth," in 18th ITS World Congress, 2011.

[6] "Bluetooth and wi-fi offer new options for travel time measurements." [Online]. Available: http://www.itsinternational.com/categories/ detection-monitoring-machine-vision/features/bluetooth-and-wi-fioffer-new-options-for-travel-time-measurements/

[7] H. Lee, H.-M. Tsai, and O. Tonguz, "On the security of intra-car wireless sensor networks," in Vehicular Technology Conference Fall (VTC 2009-Fall), 2009 IEEE 70th, Sept 2009, pp. 1-5.
[8] H.-M. Tsai, W. Viriyasitavat, O. Tonguz, C. Saraydar, T. Talty, and A. Macdonald, "Feasibility of in-car wireless sensor networks: A statistical evaluation," in Sensor, Mesh and Ad Hoc Communications and Networks, 2007. SECON '07. 4th Annual IEEE Communications Society Conference on, June 2007, pp. 101-111.

[9] U. Hoppe, R. Kessler, B. Müller, and M. Wagner, Reifendruckkontrollsysteme. Süddeutscher Verlag onpact $\mathrm{GmbH}, 2013$.

[10] M. Krstic, N. Savic, R. Kraemer, and M. Junghans, "Applying tire pressure monitoring devices for traffic management purposes," in Signals, Systems, and Electronics (ISSSE), 2012 International Symposium on, Oct 2012, pp. 1-6.

[11] I. Rouf, R. Miller, H. Mustafa, T. Taylor, S. Oh, W. Xu, M. Gruteser, W. Trappe, and I. Seskar, "Security and privacy vulnerabilities of in-car wireless networks: A tire pressure monitoring system case study," in Proceedings of the 19th USENIX Conference on Security, ser. USENIX Security'10. Berkeley, CA, USA: USENIX Association, 2010, pp. 21-21. [Online]. Available: http: //dl.acm.org/citation.cfm?id=1929820.1929848

[12] J. Schulz and M. Junghans, "Deployment of tire pressure monitoring systems for traffic monitoring and safety purposes," in 18th World Congress on Intelligent Transportation Systems, 16th-20th October, 2011.

[13] S. Cragg, "Bluetooth detection - cheap but challenging," Transport Scotland, Tech. Rep., 2012.

[14] M. Brzeska and G.-A. Chakam, "Rf modelling and characterization of a tyre pressure monitoring system," in Antennas and Propagation, 2007. EuCAP 2007. The Second European Conference on. IET, 2007, pp. $1-6$.

[15] M. Cheikh, J. David, J.-G. Tartarin, S. Kessler, and A. Morin, "Rf source characterization of tire pressure monitoring system," in Wireless Technology Conference, 2009. EuWIT 2009. European. IEEE, 2009, pp. 176-179.

[16] Y. Leng, D. Wenfeng, S. Peng, X. Ge, G. J. Nga, and S. Liu, "Study on electromagnetic wave propagation characteristics in rotating environments and its application in tire pressure monitoring," Instrumentation and Measurement, IEEE Transactions on, vol. 61, no. 6, pp. 17651777, 2012.

[17] Ettus Research Website, accessed March 2014. [Online]. Available: http://www.ettus.com

[18] GNU Radio Website, accessed March 2014. [Online]. Available: http://www.gnuradio.org

[19] "http://www.dlr.de/ts/en/desktopdefault.aspx/tabid-1237/5441_read11863/." [Online]. Available: http://www.dlr.de/ts/en/desktopdefault. aspx/tabid-1237/5441_read-11863/

[20] N. Savic, M. Junghans, and M. Krstic, "Traffic data collection using Tire Pressure Monitoring System," in Telematics - support of transport, ser. Communications in Computer and Information Science, 2014.

[21] "SUMO - Simulation of Urban MObility." [Online]. Available: http://www.sumo-sim.org/

[22] Federal Communications Commission, as of 15 April 2014. [Online]. Available: http://transition.fcc.gov/oet/ea/fccid/

[23] D. Krajzewicz, J. Erdmann, M. Behrisch, and L. Bieker, "Recent development and applications of SUMO - Simulation of Urban MObility," International Journal On Advances in Systems and Measurements, vol. 5, no. 3\&4, pp. 128-138, December 2012. [Online]. Available: http://elib.dlr.de/80483/

[24] S. Krauß, "Microscopic modeling of traffic flow: Investigation of collision free vehicle dynamics," Ph.D. dissertation, Universitat zu Koln., 1998.

[25] J. Erdmann, "Lane-Changing Model in SUMO," in Proceedings of the SUMO2014 Modeling Mobility with Open Data, May 2014.

[26] R. Oertel, "Optimierung einer auf grundlage von floating-cardatensaetzen basierenden lichtsignalanlagensteuerung," 2009.

[27] R. Oertel and P. Wagner, "Delay-time actuated traffic signal control for an isolated intersection," in 90st Annual Meeting Transportation Research Board (TRB). TRB 2011 (90th Annual Meeting), 2011.

[28] S. Breitenberger, B. Grueber, and M. Neuherz, "Extended floating car data - potenziale fuer die verkehrsinformation und notwendige durchdringungsraten," ser. Strassenverkehrstechnik, vol. 48, no. 10, 2004, pp. 522-531.

[29] F. Goessel, "Informationsentropische, spektrale und statistische untersuchungen fahrzeuggenerierter verkehrsdaten unter besonderer beruecksichtigung der auswertung und dimensionierung von fcdsystemen," Ph.D. dissertation, Dresden University of Technology., 2005. 